\title{
Using Syndromic Data for Opioid Overdose Surveillance in Utah
}

\author{
Wei Hou, Elizabeth Brutsch, Angela C. Dunn*, Cindy L. Burnett, Melissa P. Dimond and \\ Allyn K. Nakashima
}

Utah Department of Health, Salt Lake City, UT, USA

\section{Objective}

To monitor opioid-related overdose in real-time using emergency department visit data and to develop an opioid overdose surveillance report for Utah Department of Health (UDOH) and its public health partners.

\section{Introduction}

The current surveillance system for opioid-related overdoses at $\mathrm{UDOH}$ has been limited to mortality data provided by the Office of the Medical Examiner (OME). Timeliness is a major concern with OME data due to the considerable lag in its availability, often up to six months or more. To enhance opioid overdose surveillance, UDOH has implemented additional surveillance using timely syndromic data to monitor fatal and nonfatal opioid-related overdoses in Utah.

\section{Methods}

As one of the agencies participating in the National Syndromic Surveillance Program (NSSP), UDOH submits de-identified data on emergency department visit from Utah's hospitals and urgent care facilities in close to real-time to the NSSP platform. Emergency department visit data are available for analysis using the Electronic Surveillance System for the Early Notification of Community-based Epidemics (ESSENCE) system provided by NSSP. ESSENCE provides UDOH with patient-level syndromic data for analysis and early detection of abnormal patterns in emergency visits. A total of 38 out of 48 acute care hospitals and multiple urgent care facilities are enrolled in the system in Utah. More than $90 \%$ of these hospitals report chief complaint data, and discharge data are available from about $15 \%$ of the facilities. Data were analyzed by querying key terms in the chief complaint field including: any entry of: 'overdose', drug and brand names for opioids, street names, 'naloxone', and missspellings. Exclusion terms included any mention of: 'denies', 'quit', 'refill', 'withdraw', 'dependence', etc. Data containing any ICD entry of: T40.0-T40.4, T40.60, and T40.69 were included in the analysis.

\section{Results}

Between September 1, 2016 and August 31, 2017, Utah Department of Health identified 4,063 opioid-related overdose emergency department (ED) visits through the ESSENCE system using both chief complaint and discharge diagnosis queries. Of these visits, 3,865 (95\%) were identified using chief complaints alone and $198(5 \%)$ visits were added by searching the discharge diagnosis field. Opioidrelated visits comprised approximately $0.3 \%$ of the total ED visits $(1,267,244)$ reported during this time (Graph 1). More than half of the opioid-related emergency visits were reported from just five facilities. Rate of opioid-related visits ranging from 0 to 292 visits per 100,000 population per year (median: 108 visits per 100,000 population per year), with an overall rate for the state of 129 visits per100, 000 population per year. The highest rate of opioid-related visits occurred among patients aged 18 to 24 (219 visits per 100,000 population per year), and $59 \%$ of all opioid-related patients in Utah were female.

\section{Conclusions}

The results presented are estimates of opioid-related overdoses reported using close to real-time data. These results would not include visits with incomplete or incorrectly coded chief complaints or discharge codes, or cases of opioid overdose who do not present to an emergency department or urgent care facility. The results from using syndromic data are consistent with existing surveillance findings using mortality data in Utah. This suggests that syndromic surveillance data are useful for rapidly capturing opioid events, which may allow for a timelier public health response. UDOH is currently evaluating syndromic surveillance data versus hospital discharge data for opioid-related emergency department visits, which may further optimize queries in ESSENCE, in order to provide improved opioid surveillance data to local public health partners. This analysis demonstrates that using syndromic surveillance data provides a more time-efficient alternative, enabling more rapid public health interventions, which improved opportunities to reduce opioid-related morbidity and mortality in Utah.

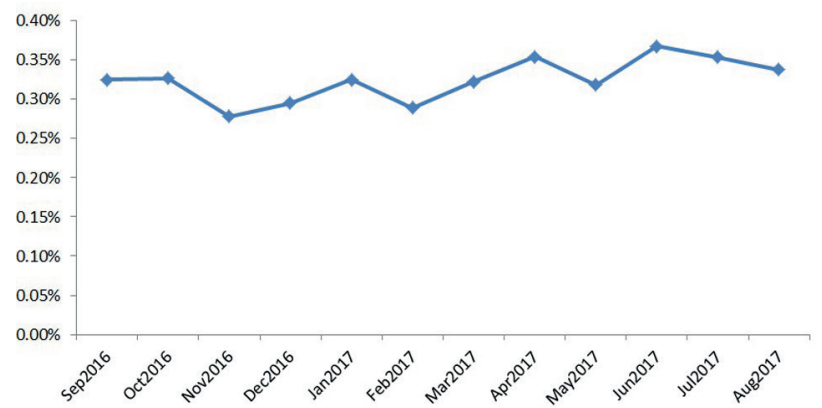

Monthly ED Visits for Opioid-related Overdose as Percent of Total Visits in Utah

\section{Keywords}

Syndromic Surveillance; Opioid Overdose; Utah; real-time surveillance

\author{
*Angela C. Dunn \\ E-mail: angeladunn@utah.gov
}

